\title{
Contribution of lexico-semantic processes to verbal short-term memory tasks: A PET activation study
}

\author{
F. Collette and S. Majerus \\ University of Liège, Belgium \\ M. Van der Linden \\ University of Liège, Belgium, and University of Geneva, Switzerland \\ P. Dabe, C. Degueldre, G. Delfiore, A. Luxen, and E. Salmon \\ University of Liège, Belgium
}

\section{Introduction}

Recent studies have demonstrated the intervention of longterm memory processes in verbal STM tasks and several cognitive models have been proposed to explain these effects. A PET study was performed in order to determine whether supplementary cerebral areas are involved when subjects have to execute short-term memory tasks for items having representations in long-term memory (in comparison to items without such representations: words vs nonwords) Results indicate that verbal STM for words specifically involves the left middle temporal gyrus (BA 21) and tempo-parietal junction (BA 39). These areas can be associated with lexical and semantic processes. These results are in agreement with cognitive models that postulate the simultaneous influence of lexical and semantic long-term representations on verbal STM processes and/or a lexico-semantic buffer.

The cerebral areas involved in the maintenance of a small amount of verbal information in memory during a brief period of time have been investigated in many positron emission tomography (PET) and functional magnetic resonance imaging (fMRI) activation experiments. These studies showed that a network of primarily left-sided brain regions was activated during the realisation of these tasks (Awh et al., 1996; Becker et al., 1994; Paulesu, Frith, \& Frackowiack, 1993; Salmon et al., 1996). These legions include the left supramarginal gyrus and Bioca's area, as well as the supplementary motor area, premotor cortex, anterior cingulate, insula, thalamus, cerebellum and para-hippocampal gyrus. The multi-component model of verbal short-term memory (STM) proposed by Baddeley and Hitch (Baddeley, 1986; Baddeley \& Hitch, 1974) was frequently used to interpret these results. This model comprises a modality-free controlling central executive that is aided by a number of subsidiary slave systems ensuring temporary maintenance of different kinds of information. Among these slave systems, the phonological loop has been the most thoroughly explored. This system is specialised for processing verbal material and is composed of two subsystems: a passive phonological input store and an articulatory rehearsal process. Numerous functional imagery studies have investigated the neural basis of the different subcomponents of the phonological loop.

In a first study, Paulesu et al. (1993) showed that activation of the supramarginal gyrus was related to the phonological store of the phonological loop, whereas activation in Broca's area was associated with the rehearsal component. These results have been confirmed by subsequent studies (e.g., Salmon et al., 1996; Schumacher et al., 1996). In a recent review paper, consisting of a finer-grained analysis of published experiments about the phonological loop, Becker, MacAndrew, and Fiez (1999) emphasised the existence of two possible foci of the phonological store within the parietal cortex: Under some experimental conditions, storage mechanisms appear in the region of the inferior supramarginal gyrus (Becker et al., 1994; Paulesu et al., 1993; Salmon et al., 1996) whereas in other studies the locus of the phonological system appears much more superior within the parietal lobe at the border of BA 
37, BA 39 and BA 40/7 (Awh et al., 1996; Smith, Jonides, \& Koeppe, 1996).

Furthermore, studies show that verbal STM tasks are influenced by representations stored in verbal long-term memory. Indeed, verbal STM span performance is larger for words than for non-words (Hulme, Roodenrys, \& Mercer, 1995; Schweickert, 1993), for high-frequency and high-imageability words than for low-frequency and low-imageability items (Bour assa \& Besner, 1994; Walker \& Hulme, 1999; Watkins \& Watkins, 1977), and also for words belonging to the same semantic category than for words from different semantic categories (Poirier \& Saint-Aubin, 1995). These data indicate that lexical and semantic information contributes to verbal STM span. In the same vein, developmental studies have shown that non-word repetition, a measure of verbal STM, is better for more wordlike non-words (Gathercole, Willis, Emslie, \& Baddeley, 1991). This wordlikeness effect suggests that immediate memory for non-words is supported by some knowledge of the phonological structure of words in a given language. Thus the influence of representations stored in longterm memory on verbal STM functioning seems to be quite a robust effect in cognitive and developmental psychological studies.

Although the neural network underlying the phonological loop processes is relatively well established at present, no functional imagery study has been specifically designed to investigate the influence of long-term memory representations on verbal STM. Indeed, some of these studies used verbal items (e.g., letters, words......) both in control and experimental tasks, whereas the remaining studies used verbal items in the experimental condition (e.g., letters) and non-verbal items in the control condition (e.g., meaningless objects). In the first case, both types of items had pre-existing long-term representations and in the second, the experimental and control tasks differed about other cognitive characteristics than the existence of pre-existing phonological or lexical long-term representations. Interestingly, Crosson et al. (1999) demonstrated that different brain regions were activated in a verbal STM task, depending on the kind of information that had to be processed. Indeed, when the short-term memory task required the retention of semantic information, left middle temporal and posterior-inferior temporal regions, inferior frontal cortex, posterior thalamus, Broca's area, and pre-SMA were activated, whereas the left extrastriate cortex and Broca's area were activated when orthographic information had to be retained. Finally, increases of cerebral activity were found in the inferior temporal-occipital junction, prefrontal cortex, pre-SMA, cerebellum, and midbrain regions when phonological information had to be maintained in verbal short-term memory. However, in the study of Crosson et al. (1999), the stimuli used in the phonological, semantic, and orthographic STM tasks were words and thus all had preexisting representations in long-term memory. Therefore, it is not possible to determine whether there is additional brain activation for the short-term stor age of stimuli that have or that do not have preexisting representations in long-term memory.

Consequently, the aim of the present study was to evaluate whether there is differential brain activation for verbal STM of items that have or do not have representations in long-term memory, in this case familiar words versus non-words. If long-term memory does support verbal STM, as predicted by empirical studies, then there should be activation of additional brain regions for the short-term retention of lists of words as compared to lists of non-words.

\section{Materials and Method}

\subsection{Subjects}

A total of 12 European right-handed volunteers ( 5 males and 7 females), aged from 18 to 30 years gave written informed consent to take part in this study, which was approved by the 
University of Liège Ethics Committee. None had any past medical history or used any medication.

\subsection{Positron emission tomography scanning}

PET data were acquired on a Siemens CTI 951 R 16/31 scanner in 3D mode. The subject's head was stabilised by a thermoplastic facemask secured to the head holder (Truscan imaging, MA), and a venous catheter was secured in a left antebrachial vein. First, a 20-min transmission scan was acquired for attenuation correction using three rotating sources of $68 \mathrm{Ge}$.. Then, regional cerebral blood flow, taken as a marker of local neuronal activity (Jueptner \& Weiller, 1995), was estimated during 12 emission scans. Each scan consisted of two frames: a 30-second background frame and a 90-second acquisition frame. The slow intravenous water $\left(\mathrm{H}_{2}{ }^{15} \mathrm{O}\right)$ infusion began 10 seconds before the second frame. Six mCi (222 $\mathrm{MBq}$ ) were injected for each scan, in $5 \mathrm{cc}$ saline, over a period of 20 seconds. The infusion was totally automated in order not to disturb the subject during the scanning period. Data were reconstructed using a Hanning filter (cutoff frequency: 05 cycle/pixel) and corrected for attenuation and background activity. The cognitive tasks were randomly distributed between subjects, with the exception that a cognitive task was not administered twice in succession.

\subsection{Cognitive tasks}

Subjects performed four different verbal repetition tasks. The tasks administered consisted of an auditory presentation of item lists that subjects had to recall aloud in serial order immediately after their presentation. Two types of items were used: words and non-words. Moreover, for each type of items, two list lengths were administered: recall of lists of one or three items. A factorial design was thus obtained that allowed exploration of the variables "memory load" (1 vs 3 items) and "pre-existing representations in long-term memory" (words vs non-words), as well as the influence of pre-existing representations in long-term memory on verbal STM functioning. Details of the different tasks are as follows:

1. One non-word condition: auditory presentation of series of one non-word that the subject had to recall immediately.

2. One word condition: auditory presentation of series of one word that the subject had to recall immediately.

3. Three non-words condition: auditory presentation of series of three non-words that the subject had to recall immediately in serial order.

4. Three words condition: auditory presentation of series of three words that the subject had to recall immediately in serial order.

A pool of 349 bisyllabic non-words was drawn up by combining French phonotactically legal syllables. From these non-words, 234 were selected in a pre-experimental phase as having a low wordlikeness with French words (for a description of the procedure, see Gathercole et al., 1991). The words used in this study were selected from the database Brulex (Content, Mousty, \& Radeau, 1990) with the following criteria: the items were bisyllabic nouns of high or medium frequency and the consonant-vowel structure of each word was closely matched to that of a non-word.

From these items three lists of one word, three words, one non-word, and three non-words were selected and were recorded on a computer, No item was repeated from one list to another. For each task, items were presented with an interval of 1 second and the response time allocated was 2 (one-item lists) or 5 (three-item lists) seconds. The three lists of each condition were randomly presented, with the restriction that two lists in the same condition were not successively presented. Subjects' responses were recorded on a tape microphone The 
recall score was termed as the number of items correctly recalled in serial order. Subjects were trained on each cognitive task before the PET session. Before each acquisition, the instructions were repeated. In order to have similar requirements in auditory processes and motor response, each task consisted of 39 items (13 series of 3 items for the three items repetition conditions, or 39 series of 1 item for the one item repetition conditions). Because of the duration of the scanning period, specific analysis of the changes of regional cerebral metabolism during encoding, storage, and retrieval were not possible.

\subsection{Data analysis}

Data were analysed using statistical parametric mapping (SPM99; Welcome Department of Cognitive Neurology, Institute of Neurology, London, UK) implemented in MATLAB. For each subject, all scans were realigned together, then normalised to a standard PET template using the same transformations (Frackowiak et al., 1997). Finally, PET images were smoothed using a Gaussian kernel of $16 \mathrm{~mm}$ full width at half maximum to accommodate inter-subject differences in gyral and functional anatomy and to suppress high-frequency noise in the images. Such transformations of the data allow for voxel by voxel averaging of data across subjects and for direct cross-reference to the anatomical features in the standard stereotactic atlas (Talairach \& Toumoux, 1988).

Differences in global activity within and between subjects were removed by analysis of covariance on a voxel by voxel basis with global count as covariate and regional activity across subjects for each task as treatment (Friston et al, 1990).. The across-task comparisons were first performed by averaging between paired measurements (fixed effect model), For each voxel in stereotactic space, the analysis of covari-ance (ANCOVA) generated a condition-specific adjusted mean $\mathrm{rCBF}$ value (normalised to $50 \mathrm{ml} / 100 \mathrm{ml} / \mathrm{min}$ ) and an associated adjusted error variance. The ANCOVA allowed the comparison of the means across conditions on a voxel by voxel basis using the $t$ statistic, The resulting sets of $t$ values constituted statistical parametric maps [SPM(t)] (Friston, Frith, Liddle, \& Frackowiak, 1991).. The $\operatorname{SPM}(\mathrm{t})$ were transformed to the unit normal distribution [SPM(Z)]. We used a SPM thresholded at $p<.001$ (voxel level), with further correction for multiple comparisons $(p<$ $.05)$. SPM was thresholded to $p<.001$ (uncorrected for multiple comparisons), when looking for activation in a region predicted from previous studies.

\section{Results}

\subsection{Cognitive performance}

Results indicate significant differences between the four conditions: the recall score in the one and three words conditions did not differ (one word: $38.87+0.24$; three words: $38.72+0.33$ ) and performances were superior to the performance in the one non-word condition $(37.74+$ $0.77)$, while more errors were found during the recall of three non-words, $29.27 \pm 0.33 ; \gamma^{2}$ ANOVA $(8,3)=34, \mathrm{p}<0001$.

\subsection{Imaging data}

\section{Conjunction analysis: Memory load.}

In order to determine the cerebral areas involved in both verbal short-term memory tasks, a conjunction analysis was performed in which the changes in cerebral activity common to the comparison of three to one items (both for words and non-words) were assessed This analysis 
showed increases of activity in the left medial frontal area (BA 8), in the anterior cingulate gyrus, in the left thalamus, and left insula. Supplementary foci of activation (non-corrected for multiple comparisons) were also found in Broca's area, in the anterior cingu-late (BA 24/32) as well as in the cerebellum bilaterally (Table 1 and Figure 1).

Conjunction analysis: type of items.

The cerebral areas specifically involved in the processing of words (with no regard to the memory load) are right-sided and include the superior temporal gyrus (BA 22) and the middle frontal gyrus (BA 9) (Table 2 and Figure 1).

Conversely, the cerebral area most involved in the processing of non-words is the right cerebellum Supplementary activation foci (non-corrected for multiple comparisons, see Table 3 and Figure 1) were found in the left cerebellum and the left superior temporal gyrus (BA 22).

TABLE 1: Conjunction analysis for the memory load

\begin{tabular}{|c|c|c|c|c|}
\hline \multirow[b]{2}{*}{ Brain area } & \multirow{2}{*}{$\begin{array}{l}\text { Stereoti } \\
\boldsymbol{X}\end{array}$} & \multirow{2}{*}{$\begin{array}{l}\text { ictic } \\
\text { coor } \\
y\end{array}$} & \multicolumn{2}{|l|}{ dinates } \\
\hline & & & $X$ & $\begin{array}{l}Z \text { - } \\
\text { score }\end{array}$ \\
\hline$p$-value $<, 001$, with & comparis & $m$ for & ltiple & pariso \\
\hline further & $c$ & $m u$ & con. & $n s$ \\
\hline $\begin{array}{l}\text { Medial frontal gyrus } \\
\text { (BA 8) }\end{array}$ & 2 & 28 & 40 & 5.76 \\
\hline $\begin{array}{l}\text { Anterior cingulate } \\
\text { gyrus } \\
\text { (BA 32) }\end{array}$ & 4 & 28 & 32 & $.4-95$ \\
\hline L thalamus & -2 & -4 & 0 & 4.77 \\
\hline $\mathrm{L}$ insula & -24 & 20 & -6 & 4.80 \\
\hline p-value к.OOl, not & $z d$ for mui & ltiple & iparito & \\
\hline correctt & & con & $n s$ & \\
\hline \multirow{2}{*}{$\begin{array}{l}\text { L infer tor frontal gyrus } \\
\text { (BA 44) }\end{array}$} & -46 & 52 & 2 & 3.13 \\
\hline & -58 & 16 & 8 & 3.13 \\
\hline \multirow[t]{5}{*}{$\mathrm{R}$ and $\mathrm{L}$ cerebellum } & 10 & -78 & -22 & 4.31 \\
\hline & 60 & -58 & -26 & 407 \\
\hline & -46 & -68 & -26 & 397 \\
\hline & -34 & -64 & -34 & 375 \\
\hline & -.44 & -74 & -32 & 3.63 \\
\hline
\end{tabular}



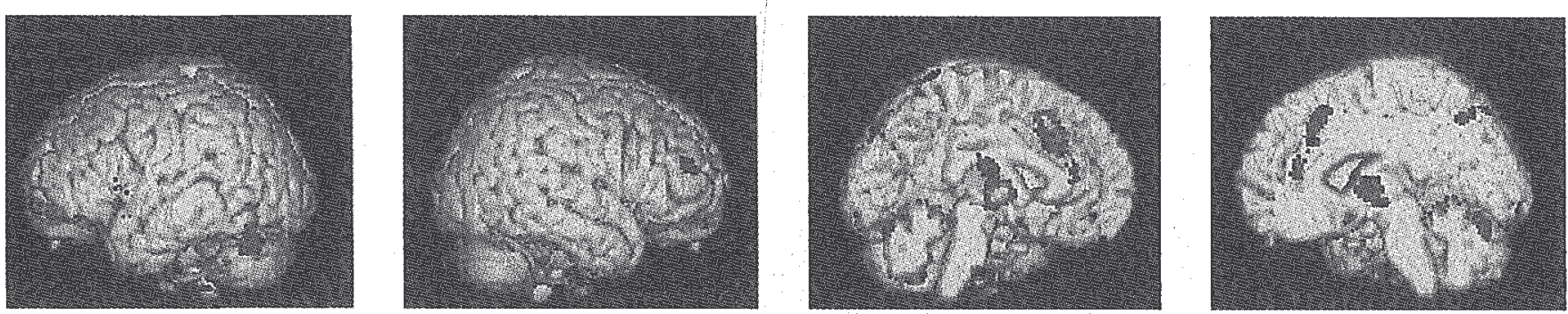

(a)
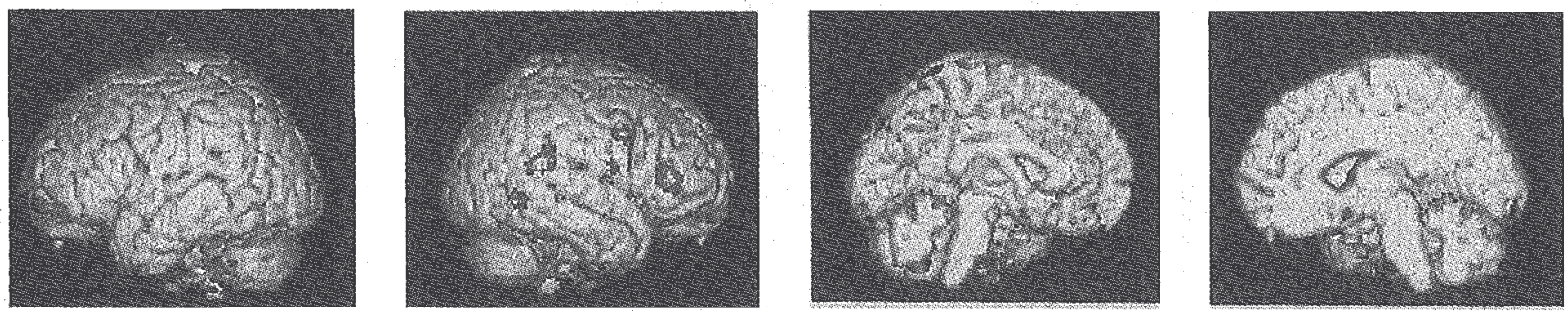

(b)
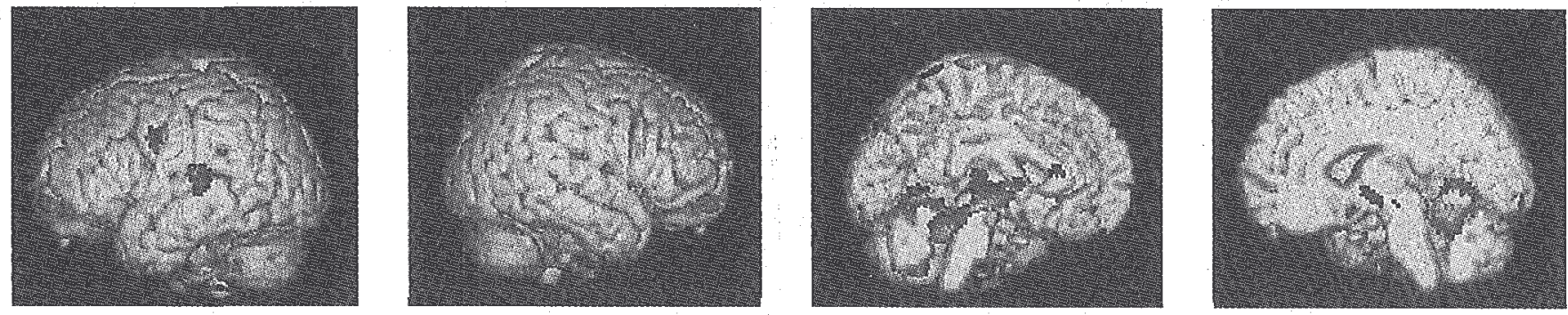

(c)
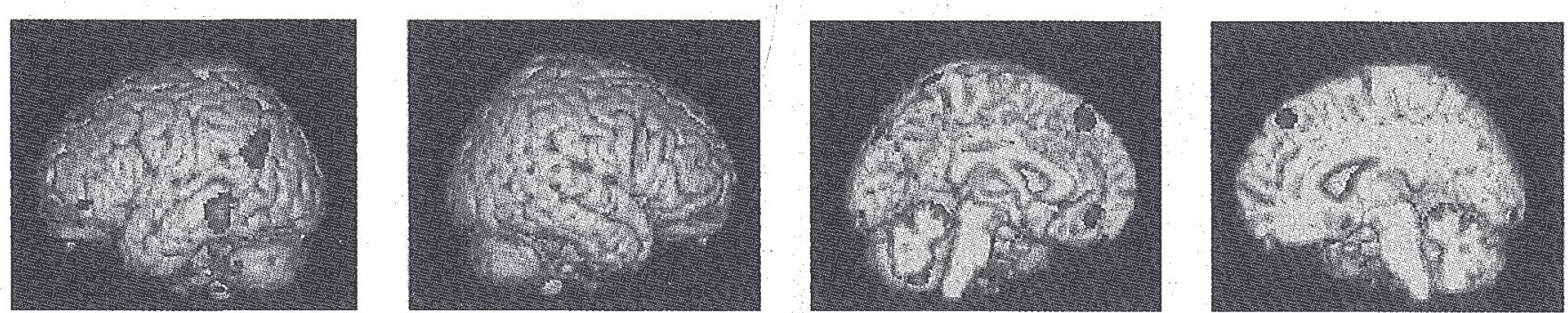

(d) 
Figure 1. Brain activation (corrected for multiple comparisons) observed (a) when the memory load for three items was compared to the memory load for one item; (b) in the processing of words in comparison to nonwords; (c) $m$ the processing of non-words in comparison to words; (d) in the interaction analysis between memory load and type of items. Coordinates of all significant regions are given respectively in Tables 1, 2, 3, and 4. Brain areas are rendered on a standard brain conforming to stereotactic space.

TABLE 2: Conjunction analysis for the item type: words-non-words

\begin{tabular}{|c|c|c|c|c|}
\hline & Ster & otactic coori & iinates & \\
\hline Brain area & $x$ & $y$ & $X$ & Z-score \\
\hline p-value $<001$, with further & \multicolumn{4}{|c|}{ corrections } \\
\hline for multiple comparisons & & & & \\
\hline \multirow{2}{*}{$\begin{array}{l}\text { R superior temporal gyrus } \\
\text { (BA 22) }\end{array}$} & 64 & -42 & 18 & 5,05 \\
\hline & 70 & -36 & 18 & 4,70 \\
\hline $\begin{array}{l}\text { R middle frontal gyrus } \\
\text { (BA 9) }\end{array}$ & 50 & 14 & 30 & 500 \\
\hline
\end{tabular}

Coordinates and Z-scores for voxels in which there was .ignificant activation foci when the processing of words was ;ompared to the processing of non-words, $\mathrm{L}=$ left hemisphere; il = right hemisphere, $x, y, z$ (in $\mathrm{mm}$ ) refer to coordinates in the falairach space (Talairach \& Tournoux, 1988)

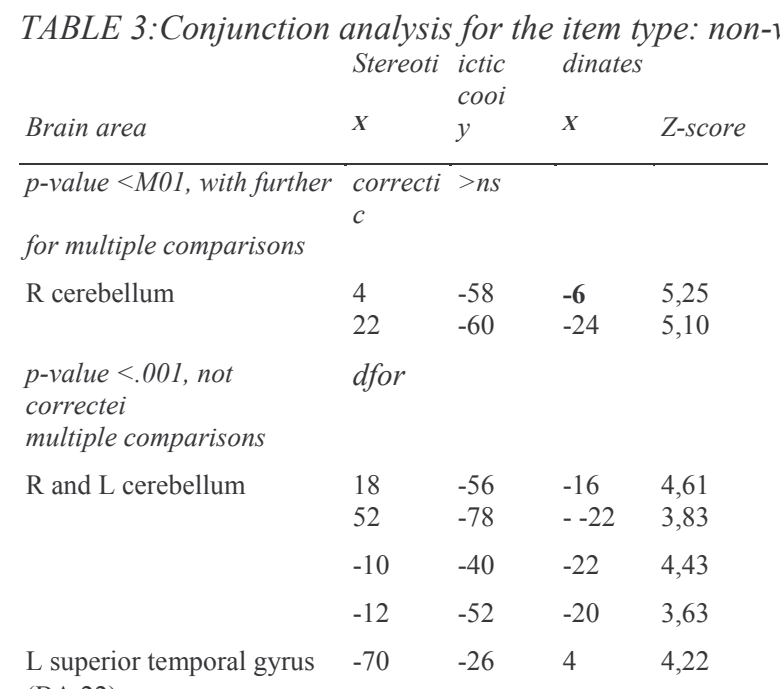

(BA 22)

Coordinates and Z-scores for voxels in which there was significant activation or deactivation foci when the processing of non-words was compared to the processing of words, $\mathrm{L}=$ left hemisphere; $\mathrm{R}$-right hemisphere, $x$, $y, z$ (in $\mathrm{mm}$ ) refer to coordinates in the Talairach space (Talairach \& Touinoux, 1988)

\section{Interaction analysis.}

Our factorial design allows us to study the effect that one variable has on the expression of the other variable, In this study, the two variables were memory load (one oi three items) and the representations in long-term memory (present or absent). The interaction between these factors identifies areas where the functioning of verbal STM varies depending on the presence or absence of pre-existing representations in long-term memory. In the present case, the influence of pre-existing representations in long-term memory on the functioning of verbal STM was assessed (see Price, Moore, \& Friston, 1997). Results showed increased rCBF specific to the storage of words in short-term memory in the left middle temporal gyrus (BA 21) and left tem-poro-parietal junction (BA 39) (see Table 4 and Figure 1). 


\begin{tabular}{lcccc}
\hline \multicolumn{5}{c}{ Stereotactic coorc linate } \\
Brain area & $*$ & $y$ & ${ }^{{ }^{1}}{ }_{X}$ & Z-score \\
\hline $\begin{array}{l}\text { o-value < 001, with further } \\
\text { for multiple comparisons }\end{array}$ & & & & \\
$\begin{array}{l}\text { L middle temporal gyrus } \\
\text { (BA 21) }\end{array}$ & -60 & -38 & -14 & 505 \\
$\begin{array}{l}\text { L temporo-parietal gyrus } \\
\text { (BA 39) }\end{array}$ & -52 & -62 & 18 & 5.00 \\
\end{tabular}

Coordinates and Z-scoies for voxels in which there was significant activation foci with an interaction analysis between the variables "memory load" and "representations in long-term memory". L = left hemisphere; $R=$ right hemisphere, $x, y, z$ (in $\mathrm{mm}$ ) refer to coordinates in the Talairach space (Talairach \& Tournoux, 1988).

\section{Discussion}

The aim of the present study was to determine whether similar cerebral areas are involved in verbal short-term memory for words and non-words, with only the former having pre-existing representations in long-term memory. We first performed two conjunction analyses in order to determine (a) cerebral areas involved in the functioning of the phonological loop and common to the two types of items (words and non-words) and (b) specific regions underlying the processing of words versus non-words, whereby the items have or have not to be stored in verbal STM, Next, the cerebral areas specific to the short-term storage of information having pre-existing long-term memory representations were isolated by interaction analysis.

When the cerebral activity in the conditions requiring recall of three words or non-words was contrasted with that found during the repetition of one word or non-word, activation foci were detected in the anterior cingulate gyrus (BA 32), medial frontal gyrus (BA 8), left insula, thalamus, and cerebellum bilaterally as well as in Br oca's area (BA 44). This network of brain areas was classically devoted in previous studies to the functional anatomy of the phonological loop (Awh et al, 1996; Paulesu et al, 1993; Salmon et al, 1996). More precisely, Paulesu et al. (1993) related the activity in Broca's area to the articu-latory rehearsal system. However, no foci of activation were found in the supramarginal gyrus, a region that has been related to phonological short-term storage of information (Paulesu et al,, 1993; Salmon et al., 1996), Recently Rypma et al., (1999) did not observe any activity in that region when verbal STM for three letters was compared to verbal STM for one letter, whereas such activity was found in the comparison of memory for six letters and for one letter. The authors interpreted these results by the existence of minimal differences in the storage requirements between three-letter and one-letter memory loads which yield similar levels of activation in the parietal cortex. Such an explanation is consistent with our results, as we have compared memory loads for three items and one item.

The cerebral areas involved in the processing of words (without regard to the memory load) are the right superior temporal gyrus (BA 22) and right middle frontal gyrus (BA 9), These results show that the processing of words, in comparison to non-words, implicates additional cerebral areas in the right hemisphere, but none in the left-sided language-processing brain regions. Many PET studies for single word processing have shown activation of bilateral temporal areas (e.g., Mummery, Ashburner, Scott, \& Wise, 1999; Petersen et al, 1989; Price et al., 1996). The fact that the right-sided increases of activity remain significant in the contr ast of word versus non-wor d suggests that only words activate language-processing areas in the right hemisphere, This is also compatible with the recent hypothesis of Pulvermüller (1999) who suggests, on the basis of electrophysiological data, that content words (nouns) in particular, as used in this study, are represented bilaterally in the brain,. On the other hand, the absence of significant activation of the left temporal areas in the word versus non-word 
contrast is probably caused by the fact that both words and non-words activate those areas. This is compatible with a recent study by Paulesu et al., (2000) who showed that non-word reading yielded even greater activation of left hemisphere areas than word reading, probably reflecting different item processing in the left and right brain region.

Surprisingly, increase of activity during the processing of words in comparison to non-words was also found in the right middle frontal gyrus (BA 9). A major distinction between words and non-words is that the former have associated semantic representations. Studies exploring the semantic representations of words showed increases of activity in prefrontal, temporal, anterior cingulate, and cerebellar regions (for a review, see Cabeza \& Nyberg, 2000).

However, prefrontal activity during semantic memory tasks has almost always been found in the left hemisphere but not in the right (e.g.., Démonet et al.,, 1992; Shaywitz et al., 1995; Thompson-Schills, D'Esposito, Aguirre, \& Far ah, 1997; War bur ton et al., 1996). We have to note that previous studies exploring the neural substrates of semantic representations used a very different methodology from that of the present study and did not require subjects to encode and retrieve lists of items. Consequently, we could suggest that the right prefrontal activation is related to the retrieval and/or monitoring of semantic information previously presented to the subject. For example, Buckner et al, (1995) demonstrated selective activation in a right prefrontal area (close to the area found in the present study) when subjects were presented with the beginning of words and asked to recall words previously studied beginning with these letters, in comparison to a task in which subjects had to produce any word beginning with these letters.

It must be emphasised that one specific cerebral area intervening in the processing of nonwords in comparison to words is the left superior temporal gyrus (BA 22). That area was classically involved in verbal tasks requiring the use of phonological representations associated to language processes (Démonet et al., 1992, 1994; Paulesu et al, 1993, 1996; Petersen et al, 1989; Sergent, Zuck, Levesque, \& McDonald, 1992), Such an increase of activity in the present study indicates that the repetition of non-words was based on a phonological network similar to that intervening in word processing. These data do not appear surprising, as the non-words were drawn up on the basis of French syllables and their structure was similar to that of words in the language. Specific foci of activation were also found in the cerebellum when subjects had to store and repeat non-words, which could be related to the necessity of considerable articulatory motor programming in the processing of these items (e.g., Paulesu et al.., 1993),

Taken as a whole, these conjunction analyses demonstrated that our results were mostly in agreement with previous studies that explored the functional network of verbal STM or the cerebral areas involved in the processing of phonological and semantic information.. Thus, this experimental design does appear relevant to exploring whether supplementary cerebral areas are involved in verbal STM for items having pie-existing long-term representations. The interaction analysis demonstrated that the left middle temporal (BA 21) gyrus and temporoparietal (BA 39) junction are specifically involved during a STM task requiring the short-term maintenance of verbal items having pre-existing long-term representations. In previous studies exploring the neural substrate of language processing, these regions have been mostly associated with lexical and semantic language representations (e.g., Binder et al., 1997; Démonet et al,.. 1992, 1994; Howard et al., 1992; Mummery, Patterson, Hodges, \& Wise, 1996; Vandenberghe et al., 1996). With reference to these studies, the increase of activity in the left temporoparietal junction in our study is in agreement with an implication of semantic processes in short-term memory for words (Demonet et al.., 1992; Mummery et al., 1996; Vandenberghe et al., 1996). Data are less clear-cut concerning the left middle temporal gyrus. Indeed, for some authors, that region would support a spoken-word lexicon (e.g., Howard et al, 1992) or phonological processes (Mazoyer et al, 1993; Zatorre, Evans, Meyer, 
\& Gjedde, 1992) while others studies have shown that this region is also involved in semantic processes (Demonet et al.., 1992). Overall, it appears that the regions that are additionally activated for the storage of words versus non-words in our study are regions mostly associated with lexical and semantic, rather than purely phonological processing, However, it is not yet possible to characterise these regions clearly with regard to their implication in auditory lexical processing (the sound-based word form) and in semantic processing (the meaning associated with the word form) (for a review concerning the intervention of these regions in cognitive processes, see Price, Indefrey, \& van Turenhout, 1999) An alternative explanation for the additional brain activation for the storage of words versus non-words is that these regions could reflect the intervention of a lexico-semantic buffer Indeed, Crosson et al. (1999) recently observed activation of the left middle temporal gyrus, close to the activation focus of our study, in a verbal STM task that required short-term retention of semantic information (in comparison to the retention -of phonological and orthographic representations). A similar activation was noted by Vandenberghe et al. (1996) during semantic judgments about words, a condition that also probably requires the intervention of temporary semantic storage. However, in the studies of Crosson et al, and Vandenberghe et al, it cannot be ruled out that the activation of the left middle temporal gyrus in the semantic working memory condition reflects the intervention of lexical and semantic language representations that support performance in the semantic working memory condition rather than a semantic buffer per se. In fact, activation of the posterior ventral temporal gyrus has also been reported in studies where the demands on a semantic buffer were minimal or non-existent, as in simple object naming tasks (A. Martin, Wiggs, Ungerleider, \& Haxby, 1996).

The results of this study can be summarised in the following way. First, the short-term memory tasks using both words and non-words involved increase of activity in Broca's area. This region was associated with the rehearsal of verbal information in the phonological loop of working memory (Baddeley, 1986). Moreover, the processing of words (but not nonwords) in short-term memory involved an increase of activity in cerebral areas involved in the storage (and/or processing) of semantic and lexical long-term knowledge or in a lexicosemantic buffer. These findings can be used to discuss the relevance of different verbal shortterm memory models, So far, we can reasonably make the assumption that these data are consistent with the existence of both a verbal STM system and long-term memory stores. But they also indicate that these systems closely interact, especially for the short-term retention of words. This is partially compatible with Baddeley's revised model of the phonological loop (Baddeley, Gathercole, \& Papagno, 1998), at least with regard to the intervention of lexical long-term representations. Indeed, their revised model postulates a close interactive relationship between the phonological store and a phonological lexicon. However, the model should be extended in order to account also for the support of semantic knowledge in verbal short-term storage tasks (see Logie, 1996, for an implication of semantic representations in verbal STM tasks). In this context, our data are also consistent with the interactive model of language and short-term memory processing of RC, Martin, Lesch, and Bartha (1999), which has the advantage of including a semantic level of processing. RC. Martin and colleagues propose that the phonological, lexical, and semantic long-term representations involved in language perception and production aie closely associated to phonological and lexicosemantic buffers. The maintenance of activation in the buffers for verbal short-term memory tasks depends on the correct feedback from the long-term language representations. In oui study, the activation of areas involved in lexical and semantic processing for the storage of words versus non-words supports this theoretical proposal. Interestingly, this model also postulates specific buffers for the temporary storage of phonological and lexical-semantic information. This is consistent with the alternative interpretation that we have given to the activation of the posterior middle temporal lobe in our study as well as with the study by 
Crosson et al, (1999). Further studies are clearly needed to determine whether activation of the posterior left middle temporal gyrus in STM tasks only reflects the intervention of lexical and semantic long-term representations or if this region also constitutes the neurobiological basis for a semantic buffer.

In conclusion, the results of the present study demonstrate that there exists a support of lexico-semantic processing during STM tasks, and that this influence comprises the intervention of a phonological lexicon and semantic long-term representations or, alternatively, of a lexico-semantic buffer,. These data allowed us to discuss the relevance of verbal STM models and demonstrate that the models that are the most in agreement with our data are those that postulate the simultaneous influence of lexical and semantic long-term representations on information-specific STMprocesses (e.g., RC. Martin et al., 1999). No activity in cerebral areas specifically devoted to phonological processes was found during the STM task for words This is probably related to the fact that the items (words and non-words) used in the different tasks had a similar phonotactic structure and were consequently based on a similar phonological network. Thus, using STM tasks in which the wordlikeness of the nonwords is systematically varied could emphasise the intervention of phonological representations specific to words and non-words in STM tasks. More generally, the present study demonstrated that neuroimaging data could be used to challenge cognitive theoretical formulations and, in the line with this study, to better specify the functional architecture of STM. For example, functional imagery techniques would be fruitful in resolving the questions of the relationships between STM and perception, or the temporal course of the different processes involved in the short-term storage of information.

\section{References}

Awh, E-, Jonides, I, Smith, E.E., Schumacher, E.H., Koeppe, RA., \& Katz, S. (1996) Dissociation of storage and ieheaisal in verbal working memory: Evidence from position emission tomography. Psychological Science, 7 , 25-31.

Baddeley, AD. (1986), Working memory, Oxford: Clarendon Press.

Baddeley, A.D., Gathercole, S,„, \& Papagno, C. (1998), The phonological loop as a language learning device Psychological Review, 105,158-173.

Baddeley, A.D., \& Hitch, GJ. (1974). Woiking memory. In G Bower (Ed.), Recent advances in learning and motivation (Vol. 8, pp. 47-90) New York: Academic Press.

Becker, JT., MacAndrew, D..K.., \& Fiez, IA. (1999). A comment on the functional localization of the phonological storage subsystem of working memoiy. Brain and Cognition, 41, 27-38.

Beckei, JT, Mintum, MA., Diehl, DJ, Dobkin, ,1, Maitidis, A, Madoff, DC, \& DeKosky, ST. (1994), Functional neuroanatomy of verbal free recall: A replication study. Human Brain Mapping, 1, 284-292.

Bindei, JR., Frost, IA, Hammeke, TA., Cox, TW., Rao, S.M., \& Piieto, T (1997). Human brain language areas identified by functional magnetic resonance imaging. Journal of Neuroscience, 17, 353-362.

Bouiassa, DC, \& Besnei, D (1994) Beyond the aiticulatory loop: A semantic contribution to serial oidei recall of subspan lists Psychonomic Bulletin \& Review, 1, 122-125.

Bucknei, RL, Peteisen, SE, Ojemann, IG, Miezin, FM., Squire, L.R., \& Raichle, ME. (1995). Functional anatomical studies of explicit and implicit memory retrieval tasks. The Journal of Neuro-sciences, 1.5, 12-29 Cabeza, R.., \& Nyberg, L. (2000). Imaging cognition II: An empirical review of 275 PET and fMRI studies. Journal of Cognitive Neuroscience, 12, 1-47. 
Content, A., Mousty, P., \& Radeau, M. (1990). BRULEX Une base de données lexicales informa-tisée pour Ie fiancais eciit et pailé. [BRULEX: A computerised lexical database for the French language], Annêe Psychologique, 90, 551-566.

Crosson, B., Rao, SM., Woodley, SL, Rosen, AC, Bobholz, J,A,,, Mayer, A.., Cunningham, IM, Hammeke, TA, Fuller, SA, Binder, JR., Cox, RW, \& Stein, EA (1999) Mapping of semantic, phonological, and orthographic veibal working memory in normal adults with functional magnetic resonance imaging. Neuropsychology, $13,171-187$.

Démonet, J-F,, Chollet, F.., Ramsay, S., Caidebat, D., Nespoulous, IL.,, Wise, R.., Rascol, A., \& Fiacko-wiak, RSJ, (1992) The anatomy of phonological and semantic processing in normal subjects. Brain, 115, 1753-1768.

Démonet, T-F., Price, CJ., Wise, R.., \& Frackowiack, RSJ (1994). Differential activation of light and left posterior cerebral regions by semantic and phonological tasks: A positron emission tomography study Neuroscience Letters, 182, 25-28.

Fiackowiak, R, Friston, K.., Frith, C, Dolan, R., \& Mazziotta, J..G. (1997). Human brain function. London: Academic Press.

Friston, KJ., Frith, CD., Liddle, PF., Dolan, RJ•.., Lammertsma, A.A., \& Frackowiak, R.S.J. (1990). The relationship between global and local changes in PET scans. Journal of Cerebral Blood Flow and Metabolism, 10, 458-466.

Friston, K.J., Frith, CD., Liddle, PF, \& Frackowiak, RSI. (1991). Comparing functional (PET) images: The assessment of significant changes. Journal of Cerebral Blood Flow and Metabolism, 11, 690-699.

Gathercole, SE., Willis, C, Emslie, H, \& Baddeley, AD (1991) The influences of number of syllables and wordlikeness on children's repetition of non-words. Applied Psycholinguistics, 12, 349-367.

Howard, D.., Patterson, K, Wise, R.., Brown, WD, Friston, KJ, Weiller, C, \& Frackowiak, R.S..J (1992). The cortical localization of the lexicons. Brain, 115,1769-1782.

Hulme, C.., Roodenrys, S.., \& Mercer, R (1995) The role of long-term memory mechanisms in memory span. British Journal of Psychology, 86, 527-536.

Jueptner, M... \& Weiller, C. (1995)., Review: Does measurement of regional cerebral blood flow reflect synaptic activity? Implications for PET and fMRI, Neurolmage, 2,148-156.

Logie, R..H. (1996). The seven ages of working memory. In M. Richards, RW. Engle, L Hasher, RH Logie, ER Stoltzfus, \& RT. Zacks (Eds.), Working memory and human cognition (pp.. 31-65). Oxford: Oxford University Press.

Martin, A, Wiggs, CL.,, Ungerleider, LG,, \& Haxby, J.V.. (1996), Neural correlates of category-specific knowledge. Nature, 379, 649-652.

Martin, RC, Lesch, M.F., \& Bartha, M..C. (1999). Independence of input and output phonology in word processing and short-term memory Journal of Memory and Language, 41, 3-29.

Mazoyer, BM, Tzourio, N., Frak, V., Syrota, A, et al. (1993) The cortical representation of speech. Journal of Cognitive Neuroscience, 5, 467-479.

Mummery, CJ.., Ashburner, J., Scott, SK, \& Wise, RJ S (1999). Functional neuroimaging of speech perception in six normal and two apfiasic subjects. Journal of the Acoustical Society of America, 106, 449-457.

Mummery, CJ, Patterson, K, Hodges, JR, \& Wise, RJS. (1996). Generating "tiger" as an animal name or a word beginning with T: Differences in brain activation. Proceedings of the Royal Society of London (B), 263, 989-995.

Paulesu, E, Frith, CD., \& Frackowiak, RSJ. (1993). The neural correlates of the verbal component of working memory. Nature, 362, 342-345. 
Paulesu, E, McCrory, E.., Fazio, F, Menoncello, L, Brunswick, N, Cappa, SF, Cotelli, M.., Cossu, G.,. Corte, F., Lorusso, M.,, Pesenti, S, Gallagher, A., Perani, D., Price, C, Frith, CD, \& Frith, U. (2000).

A cultural effect on brain function. Nature Neuro-science, 3, 91-96

Petersen, S..E., Fox, PT., Posner, M.I., Mintum, M., \& Raichle, ME (1989) Positron emission tomography studies of the processing of single words. Journal of Cognitive Neuroscience, 1,153-170.

Poirier, M., \& Saint-Aubin, J. (1995). Memory for related and unrelated words: Further evidence on the influence of semantic factors in immediate serial recall. The Quarterly Journal of Experimental Psychology, $48 A, 384-404$.

Price, CJ, Indefrey, P., \& van Turenhout, M. (1999)., The neural architecture underlying the processing of written and spoken word forms In CM. Brown \& P. Haggort (Eds.,), The neurocognition of language (pp.. 211240). Oxford: Oxford University Press.

Price, C.J., Moore, C.I, \& Friston, KJ (1997). Sub-stractions, conjunctions and interactions in experimental design of activation studies Human Brain Mapping, 5, 264-272.

Price, CJ., Wise, R..J..S., Warbuiton, EA, Moore, CJ, Howard, D, Patterson, K, Frackowiak, RSJ, \& Friston, KJ (1996) Hearing and saying The functional neuro-anatomy of auditory word processing Brain, 119, 919-931.

PuIvermullet, F (1999). Words in the brain's language Behavioral and Brain Sciences, 22, 253-336.

Rypma, B, Prabhakaran, V, Desmond, I.E., Glover, GH, \& Gabrieli, JDE (1999) Load dependent roles of frontal brain regions in the maintenance of working memory. Neuroimage, 9, 216-226.

Salmon, E., Van der Linden, M, Collette, F, Delfiore, G, Maquet, P., Degueldre, C, Luxen, A, \& Franck, G. (1996). Regional brain activity during working memory tasks Brain, 119, 1617-1625.

Schumacher, EH, Lauber, E., Awh, E, Jonides, J.,, Smith, E.E., \& Koeppe, R..A. (1996). PET evidence for an amodal verbal working memory system Neuroimage, 3, 79-88.

Schweickert, R (1993) A multinomial processing tree model for degradation and redintegration in immediate recall Memory and Cognition, 21, 168-175.

Sergent, J., Zuck, E., Levesque, M., \& MacDonald, B (1992) Position emission tomography study of letter and object processing: Empirical findings and methodological considerations. Cerebral Cortex, 2, 68-80.

Shaywitz, BA, Shaywitz, SE, Pugh, KR, Constable, RT., Skudlaiski, P., Fulbright, RK, Bionen, RA, Fletcher, J.A., Shankweller, DP, Katz, L, \& Gore, J..C (1995). Sex differences in the functional organization of the brain for language. Nature, 373, 607-609.

Smith, EE, Jonides, J, \& Koeppe, RA (1996). Dissociating verbal and spatial working memory using PET. Cerebral Cortex, 6,11-20.

Talairach, J, \& Touinoux, P, (1988). Co-planar stereotaxic atlas of the human brain: 3-dimensional proportional system: An approach to cerebral imaging Stuttgart: Thieme.

Thompson-Schill, SL, D'Esposito, M, Aguirre, GK, \& Faiah, M.J.. (1997), Role of left inferior prefrontal cortex in retrieval of semantic knowledge: A reeva-luation. Proceedings of the National Academic of Sciences, USA, 94,14792-14797.

Vandenbergh, R.., Price, C.J., Wise, RJS, Josephs, O.., \& Frackowiak, RSI (1996) Functional anatomy of a common semantic system for words and pictures. Nature, 383, 254-256.

Walker, I.., \& Hulme, C (1999), Concrete words are easier to recall than abstract words: Evidence for a semantic contribution to short-term serial recall Journal of Experimental Psychology: Learning, Memory, and Cognition, $25,1256-1271$. 
War burton, E., Wise, RJS, Price, CJ,, Weiller, C, Hadar, U,, Ramsay, S, , \& Frackowiak, R.S.J, (1996), Noun and verb retrieval by normal subjects. Studies with PET, Brain, 119, 159-179.

Watkins, O.C., \& Watkins, MJ. (1977),, Serial recall and the modality effect: Effects of word frequency. Journal of Experimental Psychology: Human Learning and Memory, 3, 712-718.

Zatorre, RJ., Evans, A..C, Meyer, E,, \& Gjedde, A, (1992) Lateralization of phonetic and pitch discrimination in speech processing Science, 256, 846-849. 